\title{
Reactant Concentrations from Fluorescence Correlation Spectroscopy with Tailored Fluorescent Probes. An Example of Local Calibration-Free $\mathrm{pH}$ Measurement.
}

\author{
Sandrine Charier, ${ }^{\dagger}$ Adrien Meglio, ${ }^{\dagger}$ Damien Alcor, ${ }^{\dagger}$ Emmanuelle Cogné-Laage, ${ }^{\dagger}$ \\ Jean-François Allemand, ${ }^{\dagger, \ddagger}$ Ludovic Jullien, ${ }^{\dagger, *}$ Annie Lemarchand ${ }^{\sharp}$ \\ † S. Charier, A. Meglio, D. Alcor, E. Cogné-Laage, L. Jullien \\ Département de Chimie (CNRS UMR 8640), École Normale Supérieure, \\ 24, rue Lhomond, 75231 Paris Cedex 05, France, \\ Fax: (+33) 1443233 25, E-mail: Ludovic.Jullien@ens.fr \\ $\ddagger$ J.-F. Allemand, \\ Département de Physique (CNRS UMR 8550), École Normale Supérieure, \\ 24, rue Lhomond, 75231 Paris Cedex 05, France \\ $\sharp$ A. Lemarchand \\ Laboratoire de Physique Théorique de la Matière Condensée (CNRS UMR 7600), \\ Université Pierre et Marie Curie, \\ 4, place Jussieu, 75252 Paris Cedex 05, France,
}

September 13, 2005

\section{Supporting Information}

- Appendix: Computation of the auto- and cross correlation functions that are relevant to the present work

- Supplementary Materials: Experimental FCS set-up (Figure 1S), Sample-containing cell that was used during the present FCS experiments (Figure 2S), Chemical process involving the smallest number of interconverting states that can be observed by FCS (Figure 3S), Evaluation of the reliability of the parameter values that can be extracted from FCS autocorrelation curves by using Eq.(9) (Figure 4S); Derivation of the intrinsic relaxation times $\tau_{i j}$ associated to the six chemical reactions displayed in Figure $7 \mathrm{~b}$ (Table 1S), Evaluation of the reliability of the parameter values that can be extracted from FCS autocorrelation curves by using Eq.(9) (Table 2S). 


\section{Appendix}

In this section, we follow the development of Krichevsky and Bonnet [1] to compute the auto- and cross correlation functions of the intensities of emission that account for the physical and chemical fluctuations occurring in the illuminated open volume $V$ supposed to contain an ideal solution of $m$ reactive components $\mathcal{A}_{i}$ (local concentrations $A_{i}(\vec{r}, t)$, average concentrations $\overline{A_{i}}=\left\langle A_{i}(\vec{r}, t)\right\rangle$, local deviations $\left.\delta A_{i}=A_{i}(\vec{r}, t)-\overline{A_{i}}\right)$.

\section{General Formalism}

At a given wavelength $\lambda$, the number of photons $n(\lambda, t)$ emitted during the sampling time $\Delta t$ from the illuminated volume $V$ is:

$$
n(\lambda, t)=\Delta t \int_{V} \sum_{i=1}^{m} \frac{\Phi_{i} \mathcal{I}_{i}(\lambda)}{\tau_{i}} A_{i}^{\star}(\vec{r}, t) d^{3} \vec{r}
$$

In Eq.(1), $\Phi_{i}, \mathcal{I}_{i}(\lambda)$ and $\tau_{i}$ respectively designate the quantum yield of fluorescence, the normalized emission spectrum $\left(\int_{0}^{\infty} \mathcal{I}_{i}(\lambda) d \lambda=1\right)$ and the lifetime of the considered excited state $\mathcal{A}_{i}^{\star}$. For longer sampling times than the typical lifetime of a singlet state (a few ns), one can assume that partial equilibrium with regards to the excitation/desexcitation process is reached for all the $\mathcal{A}$ species in every point of the illuminated volume. Indeed, excitation/desexcitation is the fastest process among the processes altering the number of photons emitted from the illuminated volume $[2,3,4]$. At low light intensities $I\left(\lambda_{e x c}, \vec{r}\right)$ (in number of photons per unit of surface and per unit of time): $A_{i}^{\star}(\vec{r}, t) \simeq \sigma_{\nu, i}\left(\lambda_{\text {exc }}\right) I\left(\lambda_{\text {exc }}, \vec{r}\right)^{\nu} \tau_{i} A_{i}(\vec{r}, t)$ where $\sigma_{\nu, i}\left(\lambda_{\text {exc }}\right)$ contains the cross section for light absorption after $\nu$-photon excitation at $\lambda_{e x c}: \sigma_{1, i}=$ $2.3 \varepsilon_{i}$ and $\sigma_{2, i}=\frac{1}{2} \delta_{2, i}$ where $\varepsilon_{i}$ and $\delta_{2, i}$ are respectively the molar absorption coefficient and the cross section for two photon absorption of $\mathcal{A}_{i}$ (after averaging on time, a similar relation holds when $I\left(\lambda_{\text {exc }}, \vec{r}\right)$ is a function of time). Under such conditions, Eq.(1) transforms into Eq.(2):

$$
n(\lambda, t)=\Delta t \int_{V} I\left(\lambda_{e x c}, \vec{r}\right)^{\nu} \sum_{i=1}^{m} Q_{i}\left(\lambda_{\text {exc }}\right) \mathcal{I}_{i}(\lambda) A_{i}(\vec{r}, t) d^{3} \vec{r}
$$

where $Q_{i}\left(\lambda_{\text {exc }}\right)=\Phi_{i} \sigma_{\nu, i}\left(\lambda_{\text {exc }}\right)$ designates the brightness of $\mathcal{A}_{i}$ at $\lambda$ upon excitation at $\lambda_{\text {exc }}$.

To reduce the shot noise and to allow for cross correlations, our FCS set-up (Figure 1S) possesses two detecting avalanche photodiodes $k$ ( $k=\mathrm{I}$ or II) that collect part of the photons emitted during $\Delta t$ :

$$
n^{k}(t)=\Delta t \int_{V} I\left(\lambda_{e x c}, \vec{r}\right)^{\nu} \sum_{i=1}^{m} \mathcal{Q}_{i}^{k}\left(\lambda_{e x c}\right) A_{i}(\vec{r}, t) d^{3} \vec{r}
$$

with

$$
\mathcal{Q}_{i}^{k}\left(\lambda_{\text {exc }}\right)=Q_{i}\left(\lambda_{\text {exc }}\right) \int_{0}^{\infty} \alpha^{k}(\lambda) \mathcal{F}^{k}(\lambda) \mathcal{E}^{k}(\lambda) \mathcal{I}_{i}(\lambda) d \lambda
$$

In Eq.(4), $\alpha^{k}(\lambda), \mathcal{F}^{k}(\lambda)$ and $\mathcal{E}^{k}(\lambda)$ respectively designate the collection efficiency of the channel $k$ at $\lambda$, the transmittance function of the optical elements located between the sample and the APD $k$, and the dependence of the APD $k$ response on $\lambda$. 
The correlation function $G^{I, I I}(\tau)$ is defined in Eq.(5):

$$
G^{I, I I}(\tau)=\frac{\left\langle\delta n^{I}\left(\left[\lambda_{1}, \lambda_{2}\right]^{I}, \vec{r}, 0\right) \delta n^{I I}\left(\left[\lambda_{1}, \lambda_{2}\right]^{I I}, \vec{r}, \tau\right)\right\rangle}{\overline{n^{I}\left(\left[\lambda_{1}, \lambda_{2}\right]^{I}, \vec{r}\right)}} \overline{n^{I I}\left(\left[\lambda_{1}, \lambda_{2}\right]^{I I}, \vec{r}\right)}
$$

in which the notation $\left[\lambda_{1}, \lambda_{2}\right]$ stands for the range of wavelengths collected by each collection channel, $I$ and $I I . G^{I, I I}(\tau)$ can be written:

$G^{I, I I}(\tau)=\frac{(\Delta t)^{2}}{\overline{n^{I}} \overline{n^{I I}}} \iint I\left(\lambda_{\text {exc }}, \vec{r}\right)^{\nu} I\left(\lambda_{\text {exc }}, \vec{r}^{\prime}\right)^{\nu} \sum_{i, j} \mathcal{Q}_{i}^{I}\left(\lambda_{\text {exc }}\right) \mathcal{Q}_{j}^{I I}\left(\lambda_{\text {exc }}\right)\left\langle\delta A_{i}(\vec{r}, 0) \delta A_{j}\left(\vec{r}^{\prime}, \tau\right)\right\rangle d^{3} \vec{r} d^{3} \vec{r}^{\prime}$

$G^{I, I I}(\tau)$ is a convolution of the auto- and cross-correlation functions of the concentration fluctuations with the excitation profile.

In Eq.(6), $\left\langle\delta A_{i}(\vec{r}, 0) \delta A_{j}\left(\vec{r}^{\prime}, \tau\right)\right\rangle$ can be calculated as soon as $\delta A_{j}(\vec{r}, \tau)$ is known as a function of the initial conditions $\delta A_{j}(\vec{r}, 0)$. Indeed, the ideality of the solution imposes:

$$
\left\langle\delta A_{i}(\vec{r}, 0) \delta A_{j}\left(\vec{r}^{\prime}, 0\right)\right\rangle=\overline{A_{i}} \delta_{i j} \delta\left(\vec{r}-\vec{r}^{\prime}\right)
$$

The dependence of $\delta A_{j}(\vec{r}, \tau)$ on $\delta A_{j}(\vec{r}, 0)$ is obtained from solving Eq.(8):

$$
\frac{\partial \delta A_{i}(\vec{r}, t)}{\partial t}=D_{i} \nabla^{2} \delta A_{i}(\vec{r}, t)+\sum_{j=1}^{m} \kappa_{i j} \delta A_{j}(\vec{r}, t)
$$

In Eq.(8), the first term refers to the concentration changes resulting from diffusion (diffusion coefficient $D_{i}$ ) whereas the second linearized term is related to concentration changes close to equilibrium originating from occurrences of chemical reactions (the $\kappa_{i j}$ coefficients of the matrix $\mathbf{K}=\left[\kappa_{i j}\right]$ are combined from the rate constants of the chemical reactions, and the $\overline{A_{i}}$ ). Eq.(8) is solved after Fourier transform leading to Eq.(9):

$$
\frac{\partial \widetilde{A_{i}}(\vec{q}, t)}{\partial t}=\sum_{j=1}^{m} M_{i j} \widetilde{A_{j}}(\vec{q}, t)
$$

where $\widetilde{A_{i}}(\vec{q}, t)=(2 \pi)^{(-3 / 2)} \int \exp (i \vec{q} \vec{r}) \delta A_{i}(\vec{r}, t) d^{3} \vec{r}$ and $M_{i j}=\kappa_{i j}-D_{i} q^{2} \delta_{i j}$. Introducing the eigenvalues $\lambda^{(s)}$ and the eigenvectors $X^{(s)}$ of the matrix $\mathbf{M}=\left[M_{i j}\right]$, and the inverse matrix $\mathbf{X}^{-1}$ of the eigenvectors $X^{(s)}$, Eq.(6) eventually provides [1]:

$$
G^{I, I I}(\tau)=\frac{(2 \pi)^{-3}}{\left(\sum_{i=1}^{m} \mathcal{Q}_{i}^{I} \overline{A_{i}}\right)\left(\sum_{j=1}^{m} \mathcal{Q}_{j}^{I I} \overline{A_{j}}\right)} \int_{V} e^{\left[-\frac{\omega_{x y}^{2}}{4}\left(q_{x}^{2}+q_{y}^{2}\right)-\frac{\omega_{z}^{2}}{4} q_{z}^{2}\right]} \sum_{i, j} H_{i j} d^{3} \vec{q}
$$

with

$$
H_{i j}=\mathcal{Q}_{i}^{I}\left(\lambda_{e x c}\right) \mathcal{Q}_{j}^{I I}\left(\lambda_{e x c}\right) \overline{A_{i}} \sum_{s} X_{j}^{(s)} \exp \left(\lambda^{(s)} \tau\right)\left(X^{-1}\right)_{i}^{(s)} d^{3} \vec{q}
$$

In Eq.(10), one assumes that the illumination intensity profile is 3D-Gaussian:

$$
I(\vec{r}, t)=I_{0} \exp \left[-\frac{2\left(x^{2}+y^{2}\right)}{\omega_{x y}^{2}}-\frac{2 z^{2}}{\omega_{z}^{2}}\right]
$$

where $\omega_{z}$ and $\omega_{x y}$ are the sizes of the beam waist in the direction of the propagation of light and in the perpendicular direction respectively.

The final expression of $G^{I, I I}(\tau)$ can be obtained by determining the $\vec{q}$-dependence of $H_{i j}$ for each particular case. 


\section{Isomerization coupled with diffusion}

The chemical processes involving the smallest number of interconverting states that can be observed by FCS are displayed in Figure 3S: An isomerization occurs between the ground states $\mathcal{A}_{1}$ and $\mathcal{A}_{2}$, and between the excited states $\mathcal{A}_{1}^{\star}$ and $\mathcal{A}_{2}^{\star}$. In the present case, Eq.(8) becomes:

$$
\begin{aligned}
& \frac{\partial \delta A_{1}(\vec{r}, t)}{\partial t}=-\left(k_{12}+k_{11^{\star}}\right) \delta A_{1}(\vec{r}, t)+k_{21} \delta A_{2}(\vec{r}, t)+k_{1^{\star} 1} \delta A_{1^{\star}}(\vec{r}, t)+D_{1} \Delta \delta A_{1}(\vec{r}, t) \\
& \frac{\partial \delta A_{2}(\vec{r}, t)}{\partial t}=k_{12} \delta A_{1}(\vec{r}, t)-\left(k_{21}+k_{22^{\star}}\right) \delta A_{2}(\vec{r}, t)+k_{2^{\star} 2_{2}} \delta A_{2^{\star}}(\vec{r}, t)+D_{2} \Delta \delta A_{2}(\vec{r}, t) \\
& \frac{\partial \delta A_{1^{\star}}(\vec{r}, t)}{\partial t}=k_{11^{\star}} \delta A_{1}(\vec{r}, t)-\left(k_{1^{\star} 2^{\star}}+k_{1^{\star} 1}\right) \delta A_{1^{\star}}(\vec{r}, t)+k_{2^{\star} 1^{\star}} \delta A_{2^{\star}}(\vec{r}, t)+D_{1^{\star}} \Delta \delta A_{1^{\star}}(\vec{r}, t) \\
& \frac{\partial \delta A_{2^{\star}}(\vec{r}, t)}{\partial t}=k_{22^{\star}} \delta A_{2}(\vec{r}, t)+k_{1^{\star} 2^{\star}} \delta A_{1^{\star}}(\vec{r}, t)-\left(k_{2^{\star} 1^{\star}}+k_{2^{\star}}\right) \delta A_{2^{\star}}(\vec{r}, t)+D_{2^{\star}} \Delta \delta A_{2^{\star}}(\vec{r}, t)
\end{aligned}
$$

Isomerization as well as excitations occur without major change of molecular volume leading the diffusion coefficients unchanged [5]. Thus it is reasonable to assume that $D_{1}=D_{1^{\star}}=D_{2}=D_{2^{\star}}=d$.

\section{The reactivity of the ground state is observed by FCS}

The intrinsic relaxation times $\tau_{11^{\star}}=\left(k_{11^{\star}}+k_{1^{\star} 1}\right)^{-1}$ and $\tau_{22^{\star}}=\left(k_{22^{\star}}+k_{2^{\star}}\right)^{-1}$ associated to the excitation/desexcitation processes lie in the ns range (typical lifetimes of the singlet excited states $\mathcal{A}$ and $\mathcal{A}_{2}^{\star}$ ). In most chemical circumstances, $\tau_{11^{\star}}$ and $\tau_{22^{\star}}$ are much smaller than the intrinsic relaxation times $\tau_{12}=\left(k_{12}+k_{21}\right)^{-1}$ and $\tau_{1^{\star} 2^{\star}}=\left(k_{1^{\star} 2^{\star}}+k_{2^{\star} 1^{\star}}\right)^{-1}$ associated to the isomerizations. For longer sampling times than the typical lifetime of $\mathcal{A}_{1}^{\star}$ and $\mathcal{A}_{2}^{\star}$, one can thus assume that partial equilibrium with regards to the excitation/desexcitation processes is reached in every point of the illuminated volume: $\frac{\overline{A_{1}^{\star}}}{\overline{A_{1}}} \simeq K_{11^{\star}}=\frac{k_{11^{\star}}}{k_{1^{\star} 1}}$ and $\frac{\overline{A_{2}^{\star}}}{\overline{A_{2}}} \simeq K_{22^{\star}}=\frac{k_{22^{\star}}}{k_{2^{\star} 2}}$. Then the mechanism (13-16) reduces to Eq.(17) by summing Eqs.(13) and (15), and Eqs.(14) and (16):

$$
\left\{\frac{1}{1+K_{11^{*}}} \mathcal{A}_{1}, \frac{K_{11^{*}}}{1+K_{11^{*}}} \mathcal{A}_{1}^{\star}\right\} \quad \underset{\kappa_{21}}{\rightleftharpoons} \quad\left\{\frac{1}{1+K_{22^{*}}} \mathcal{A}_{2}, \frac{K_{22^{*}}}{1+K_{22^{*}}} \mathcal{A}_{2}^{\star}\right\}
$$

with

$$
\begin{aligned}
& \kappa_{12}=\frac{1}{1+K_{11^{*}}} k_{12}+\frac{K_{11^{*}}}{1+K_{11^{*}}} k_{1^{*} 2^{*}} \\
& \kappa_{21}=\frac{1}{1+K_{22^{*}}} k_{21}+\frac{K_{22^{*}}}{1+K_{22^{*}}} k_{2^{*} 1^{*}}
\end{aligned}
$$

In the present investigation, the typical values of the laser intensities $I\left(\lambda_{e x c}, \vec{r}\right)$ and of the cross sections for two-photon absorption $\delta_{2}\left(\lambda_{\text {exc }}\right)$ are such that $K_{11^{*}} \ll 1$ et $K_{22^{*}} \ll 1[6]$. Then $\left\{\frac{1}{1+K_{11^{*}}} \mathcal{A}_{1}, \frac{K_{11^{*}}}{1+K_{11^{*}}} \mathcal{A}_{1}^{\star}\right\} \approx$ $\mathcal{A}_{1}$ and $\left\{\frac{1}{1+K_{22^{*}}} \mathcal{A}_{2}, \frac{K_{22^{*}}}{1+K_{22^{*}}} \mathcal{A}_{2}^{\star}\right\} \approx \mathcal{A}_{2}$ :

$$
\begin{aligned}
& \kappa_{12} \approx k_{12} \\
& \kappa_{21} \approx k_{21}
\end{aligned}
$$

Thus, although relying on the analyses of the fluctuations of the fluorescence intensity from the excited states $\mathcal{A}_{1}^{\star}$ et $\mathcal{A}_{2}^{\star}$, FCS reveals the reactivity of the ground states in most encountered experimental situations. This is especially significant since the rate constants of chemical reactions can be sometimes 
considerably altered by the nature, ground or excited, of the state (acid-base reactions for instance) $[7,8,9]$.

Expression of the correlation function $G^{I, I I}(\tau)$

Eq.(17) is now rewritten:

$$
\mathcal{A}_{1} \stackrel{\kappa_{12}}{\rightleftharpoons} \underset{\kappa_{21}}{\rightleftharpoons} \quad \mathcal{A}_{2}
$$

keeping in mind that $\mathcal{A}_{1}$ and $\mathcal{A}_{2}$ are averaged species involving the ground and the excited states.

Eq.(8) is written here:

$$
\begin{aligned}
& \frac{\partial \delta A_{1}(\vec{r}, t)}{\partial t}=-\kappa_{12} \delta A_{1}(\vec{r}, t)+\kappa_{21} \delta A_{2}(\vec{r}, t)+d \Delta \delta A_{1}(\vec{r}, t) \\
& \frac{\partial \delta A_{2}(\vec{r}, t)}{\partial t}=\kappa_{12} \delta A_{1}(\vec{r}, t)-\kappa_{21} \delta A_{2}(\vec{r}, t)+d \Delta \delta A_{2}(\vec{r}, t)
\end{aligned}
$$

and the matrix $\mathbf{M}$ associated to $(13,14)$ :

$$
\mathbf{M}=\left[\begin{array}{cc}
-\left(\kappa_{12}+d q^{2}\right) & \kappa_{21} \\
\kappa_{12} & -\left(\kappa_{21}+d q^{2}\right)
\end{array}\right]
$$

whose eigenvalues are:

$$
\begin{aligned}
& \lambda^{(1)}=-d q^{2} \\
& \lambda^{(2)}=-R_{12}-d q^{2}
\end{aligned}
$$

with $R_{12}=\kappa_{12}+\kappa_{21}$. The eigenvalue $\lambda^{(1)}$ reveals the conservation law during the chemical reaction (22); then only diffusion can alter the density in $\mathcal{A}_{1}$ and $\mathcal{A}_{2} . \lambda^{(2)}$ additionally contains the relaxation towards chemical equilibrium following a fluctuation of the concentrations in $\mathcal{A}_{1}$ and $\mathcal{A}_{2}$ due to occurrence of reaction (22).

Introducing the apparent equilibrium constant $K_{12}=\frac{\kappa_{12}}{\kappa_{21}}$ and the total concentration $C=\overline{A_{1}}+\overline{A_{2}}$, one obtains:

$$
\begin{aligned}
& H_{11}=\frac{\mathcal{Q}_{1}^{I} \mathcal{Q}_{1}^{I I}{\overline{A_{1}}}^{2}}{C}\left(1+K_{12} e^{-R_{12} t}\right) e^{-d q^{2} t} \\
& H_{22}=\frac{\mathcal{Q}_{2}^{I} \mathcal{Q}_{2}^{I I} \bar{A}_{2}^{2}}{C}\left(1+\frac{1}{K_{12}} e^{-R_{12} t}\right) e^{-d q^{2} t} \\
& H_{12}=\frac{\mathcal{Q}_{1}^{I} \mathcal{Q}_{2}^{I I} \overline{A_{1}} \overline{A_{2}}}{C}\left(1-e^{-R_{12} t}\right) e^{-d q^{2} t} \\
& H_{21}=\frac{\mathcal{Q}_{2}^{I} \mathcal{Q}_{1}^{I I} \overline{A_{1}} \overline{A_{2}}}{C}\left(1-e^{-R_{12} t}\right) e^{-d q^{2} t}
\end{aligned}
$$

and:

$$
\begin{aligned}
\sum_{i, j} H_{i j}= & \frac{\left(\mathcal{Q}_{1}^{I} \overline{A_{1}}+\mathcal{Q}_{2}^{I} \overline{A_{2}}\right)\left(\mathcal{Q}_{1}^{I I} \overline{A_{1}}+\mathcal{Q}_{2}^{I I} \overline{A_{2}}\right)}{C} \\
& \times\left[1+K_{12}\left(\frac{Q^{I}-1}{Q^{I}+K_{12}}\right)\left(\frac{Q^{I I}-1}{Q^{I I}+K_{12}}\right) e^{-R_{12} t}\right] e^{-d q^{2} t}
\end{aligned}
$$




$$
Q^{k}=\frac{\mathcal{Q}_{1}^{k}}{\mathcal{Q}_{2}^{k}}
$$

leading to the final expression of the correlation function:

$$
\begin{aligned}
G^{I, I I}(\tau)= & \frac{1}{\bar{N}}\left(1+\frac{\tau}{\tau_{D}}\right)^{-1}\left(1+\frac{\tau}{\omega^{2} \tau_{D}}\right)^{-1 / 2} \\
& \times\left[1+K_{12}\left(\frac{Q^{I}-1}{Q^{I}+K_{12}}\right)\left(\frac{Q^{I I}-1}{Q^{I I}+K_{12}}\right) e^{-R_{12} t}\right]
\end{aligned}
$$

where $\bar{N}$ is the average number of molecules contained in the illuminated volume, $T_{D}=\frac{\omega_{x y}^{2}}{4 d \nu}$ and $\omega=$ $\frac{\omega_{z}}{\omega_{x y}}$.

In this particular case where the chemical reaction is assumed not to change the diffusion properties, $G^{I, I I}(\tau)$ is the product of the diffusive term and of the term in square brackets associated to the chemical relaxation. For $\tau \ll \frac{1}{R_{12}}$ :

$$
G^{I, I I}(\tau) \approx \frac{\mathcal{Q}_{1}^{I} \mathcal{Q}_{1}^{I I} \overline{N_{1}}+\mathcal{Q}_{2}^{I} \mathcal{Q}_{2}^{I I} \overline{N_{2}}}{\left(\mathcal{Q}_{1}^{I} \overline{N_{1}}+\mathcal{Q}_{2}^{I \overline{N_{2}}}\right)\left(\mathcal{Q}_{1}^{I I} \overline{N_{1}}+\mathcal{Q}_{2}^{I I} \overline{N_{2}}\right)}
$$

where $\overline{N_{1}}$ and $\overline{N_{2}}$ respectively designate the average numbers of molecules of $\mathcal{A}_{1}$ and $\mathcal{A}_{2}$ contained in the illuminated volume. At such short times, the reactive mixture of $\mathcal{A}_{1}$ and $\mathcal{A}_{2}$ essentially behaves as a nonreactive mixture of two fluorescent species. After the relaxation time $\tau_{12}=\frac{1}{R_{12}}$ of the reaction (22), the chemical reaction does not contribute anymore to the correlation function: In this kinetic regime, $\mathcal{A}_{1}$ and $\mathcal{A}_{2}$ are in "fast" exchange and the system apparently contains only one virtual species $\left\{\mathcal{A}, \mathcal{A}_{2}\right\}$ associated to the diffusion coefficient $d$.

\section{One chemical reaction coupled to diffusion; three species}

One is now interested in the more general situation encountered in chemistry where three species are involved in the reaction (1). In relation with the present purpose, $\mathcal{H}$ designates the reactant of interest whereas $\left\{\mathcal{A}_{1}, \mathcal{A}_{2}\right\}$ is the $\mathcal{H}$-specific probe exchanging between two states of different brightness. In line with the present series of experiments, we consider that:

- the diffusion coefficients of $\mathcal{A}_{1}$ and $\mathcal{A}_{2}$ are identical and equal to $d$. In contrast, the diffusion coefficient of the third reactant $\mathcal{H}$ is equal to $D$.

- $\mathcal{H}$ is not fluorescent $\left(\mathcal{Q}_{\mathcal{H}}^{k}=0\right)$.

One introduces the equilibrium constant $K_{12}=\frac{k_{12}}{k_{21}}$ and the total concentration in $\mathcal{A}_{1}$ and $\mathcal{A}_{2}: C=$ $\overline{A_{1}}+\overline{A_{2}}$.

Eq.(8) here becomes:

$$
\begin{aligned}
& \frac{\partial \delta A_{1}(\vec{r}, t)}{\partial t}=-\kappa_{12} \delta A_{1}(\vec{r}, t)+\kappa_{21} \delta A_{2}(\vec{r}, t)+k \delta H(\vec{r}, t)+d \Delta \delta A_{1}(\vec{r}, t) \\
& \frac{\partial \delta A_{2}(\vec{r}, t)}{\partial t}=\kappa_{12} \delta A_{1}(\vec{r}, t)-\kappa_{21} \delta A_{2}(\vec{r}, t)-k \delta H(\vec{r}, t)+d \Delta \delta A_{2}(\vec{r}, t) \\
& \frac{\partial \delta H(\vec{r}, t)}{\partial t}=\kappa_{12} \delta A_{1}(\vec{r}, t)-\kappa_{21} \delta A_{2}(\vec{r}, t)-k \delta H(\vec{r}, t)+D \Delta \delta H(\vec{r}, t)
\end{aligned}
$$




$$
\begin{aligned}
\kappa_{12} & =k_{12} \\
\kappa_{21} & =k_{21} \bar{H} \\
k & =k_{21} \overline{A_{2}}
\end{aligned}
$$

and the matrix $\mathbf{M}$ associated to (35-37):

$$
\mathbf{M}=\left[\begin{array}{ccc}
-\left(\kappa_{12}+d q^{2}\right) & \kappa_{21} & k \\
\kappa_{12} & -\left(\kappa_{21}+d q^{2}\right) & -k \\
\kappa_{12} & -\kappa_{21} & -\left(k+D q^{2}\right)
\end{array}\right]
$$

the eigenvalues of which are:

$$
\begin{aligned}
\lambda^{(1)}= & -d q^{2} \\
\lambda^{(2)}= & -\frac{1}{2}\left[\kappa_{12}+\kappa_{21}+k+(d+D) q^{2}\right] \\
& +\frac{1}{2} \sqrt{\left[\left(\kappa_{12}+\kappa_{21}+k\right)+(d-D) q^{2}\right]^{2}+4 k(D-d) q^{2}} \\
\lambda^{(3)}= & -\frac{1}{2}\left[\kappa_{12}+\kappa_{21}+k+(d+D) q^{2}\right] \\
& -\frac{1}{2} \sqrt{\left[\left(\kappa_{12}+\kappa_{21}+k\right)+(d-D) q^{2}\right]^{2}+4 k(D-d) q^{2}}
\end{aligned}
$$

In the general case, the preceding expressions do not lead to a simple analytical expression for the correlation function $G^{I, I I}(\tau)$. Nevertheless, an approximate analytical expression can be obtained in two limiting cases.

The relaxation time $\tau_{12}$ associated to the chemical reaction is much smaller than the diffusion time $\tau_{D}$

In their pioneering work involving complexation of ethidum bromide by DNA, Magde, Elson and Webb developed the expressions of the eigenvalues (41-43) taking $\frac{\tau_{12}}{\tau_{D}}$ as a small term [10,11,12]. Lamb et al revisited and extended these calculations [13]. They also performed a series of experiments in which the corresponding approximation was relevant $[13,14]$.

\section{The concentration of $\mathcal{A}_{1}$ and $\mathcal{A}_{2}$ is much less than the concentration of $\mathcal{H}$}

The availability of sensitive detectors (APD for instance) allowed to envisage a more favorable approximation than the latter. Provided that $k \ll \kappa_{12}+\kappa_{21}$, the second term under the square root in the expressions of $\lambda^{(2)}$ and $\lambda^{(3)}$ can be neglected in front of the first. Then:

$$
\begin{aligned}
& \lambda^{(1)}=-d q^{2} \\
& \lambda^{(2)}=-D q^{2} \\
& \lambda^{(3)}=-R_{12}-d q^{2}
\end{aligned}
$$

with $R_{12}=\kappa_{12}+\kappa_{21}$. The condition $k \ll \kappa_{12}+\kappa_{21}$ is noticeably fulfilled as soon as $C \ll \bar{H}$ [13]. 
The occurrence of a chemical reaction (1) is naturally coupled to the diffusion of the states $\mathcal{A}$ and $\mathcal{A}_{2}$. For instance, $\mathcal{A}_{1}$ becomes in local excess and $\mathcal{A}_{2}$ is locally lacking with regard to the external reservoir when reaction (1) takes place forward. Then relaxation is obtained by diffusion of $\mathcal{A}_{1}$ and $\mathcal{A}_{2}$. $\mathcal{H}$ is either produced or consumed during the same reactive event and the reaction (1) is similarly coupled to the $\mathcal{H}$ diffusion. Nevertheless, the occurrence of reaction (1) does not modify $\bar{H}$ when $C \ll \bar{H}$. Under such conditions, the concentration $\bar{H}$ is independent on the occurrences of the conversions between the $\mathcal{A}_{1}$ and $\mathcal{A}_{2}$ states and the reaction (1) becomes equivalent to the reaction (22). Indeed, the expressions of $\lambda^{(1)}$ and $\lambda^{(3)}$ here identify to the expressions (25) and (26). The additional eigenvalue $\lambda^{(2)}$ is associated to the diffusion of $\mathcal{H}$.

If $\mathcal{Q}_{\mathcal{H}}=0$, the different expressions obtained in the isomerization case apply to the more general situation addressed in this subsection by substituting the apparent isomerization constant $\mathcal{K}_{12}=\frac{K_{12}}{\bar{H}}$ to $K_{12}$ in Eqs.(27-33). When the channels $I$ and $I I$ share identical optical elements, the cross correlation function $G^{I, I I}(\tau)$ is equal to the autocorrelation function of the fluorescence emission from the illuminated volume $V, G(\tau)$, given in Eq.(3). 


\section{Supplementary Materials}

Figures

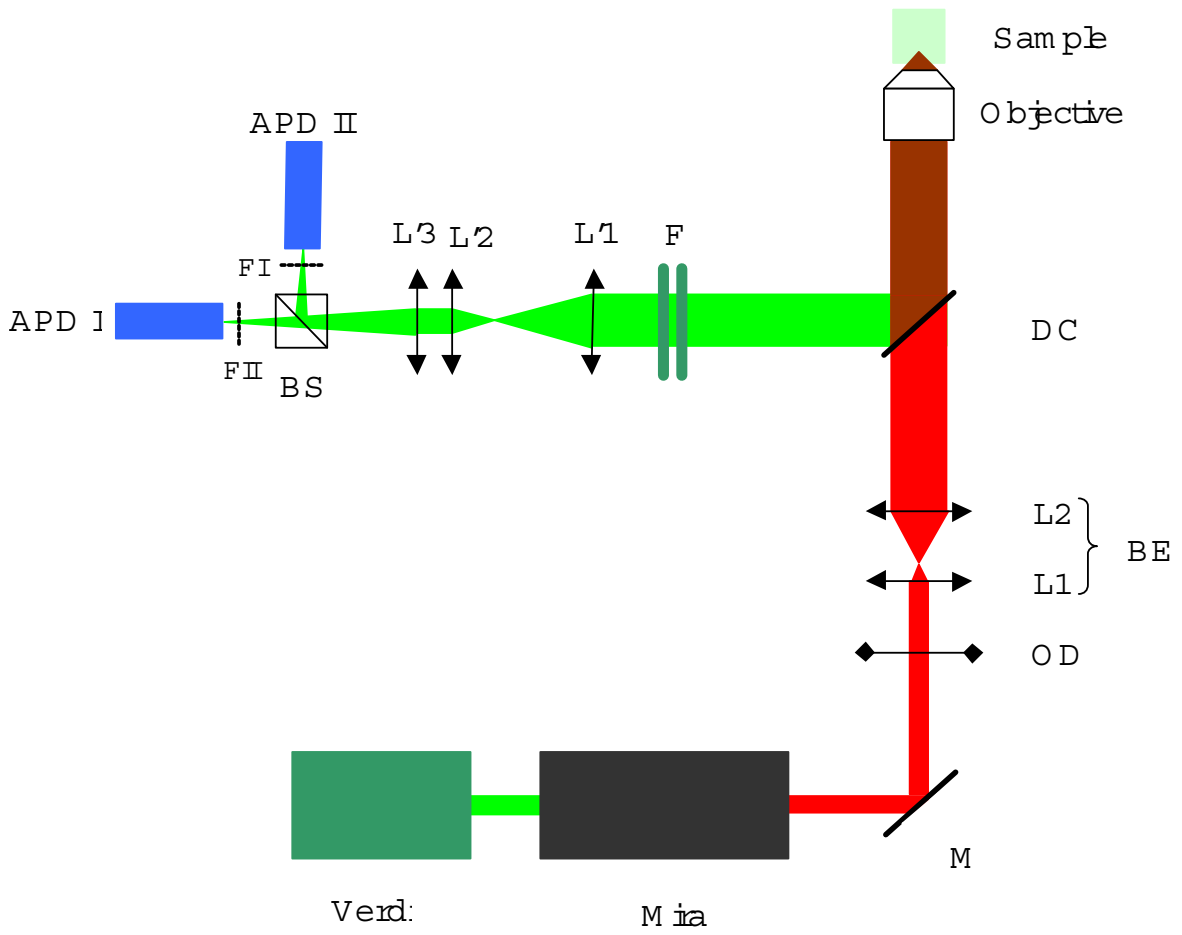

Figure 1S: FCS set-up relying on two-photon excitation that was used to perform the present series of experiments. Experiments were performed with a mode-locked femtosecond Ti:sapphire laser (Mira) pumped by a diode laser (Verdi) that delivers photon pulses at $76 \mathrm{MHz}$ repetition rate in the 700-1000 nm range. $M$ : mirror; OD: optical density; $L 1, L 2$ : focusing lenses; $B E$ : beam expander; $D C$ : dichroic mirror; $F$ : short-pass filter; $L^{\prime} 1, L^{\prime} 2, L^{\prime} 3$ : focusing lenses; $B S$ : 50/50 splitting cube; FI, FII: bandpass filters; $A P D I, A P D I I$ : avalanche photodiodes. The incoming beam is focused in the observed solution. Due to two-photon excitation that quadratically depends on light intensity, fluorescence emission is only significant from a tiny volume approximated as 3D-Gaussian in the micrometer range. Fluorescence is collected by the objective and the intensities measured by the avalanche photodiodes are cross correlated. 


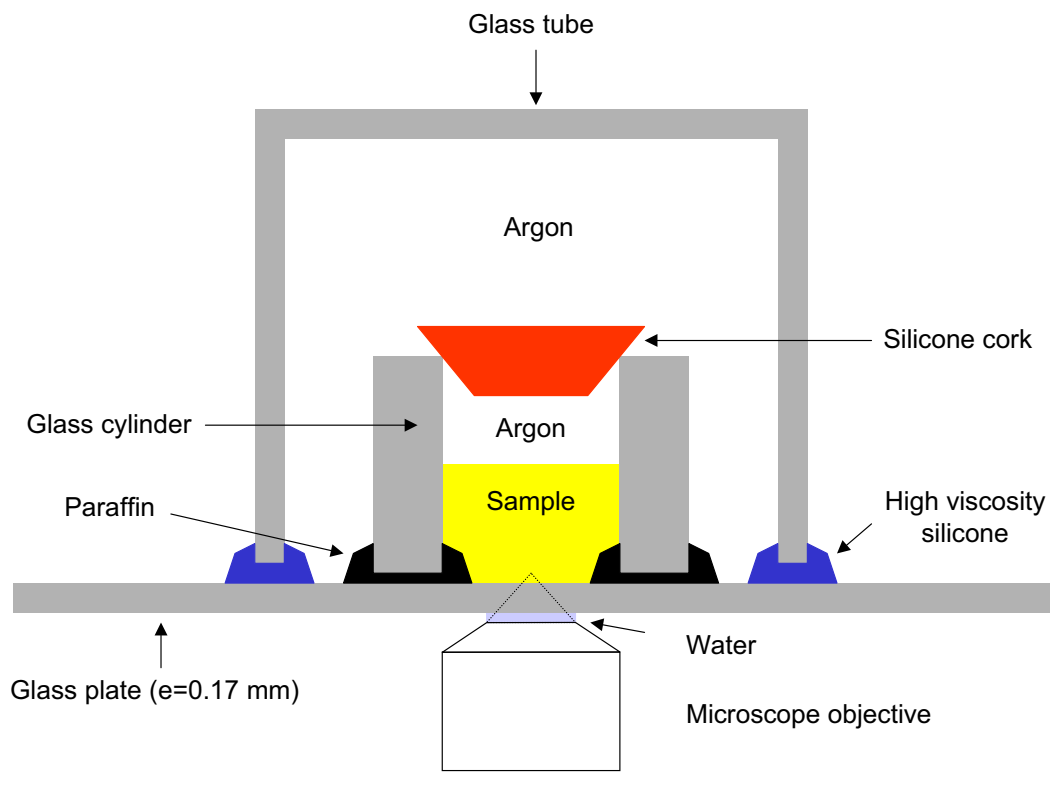

Figure 2S. Sample-containing cell under argon that was used during the present FCS experiments. 


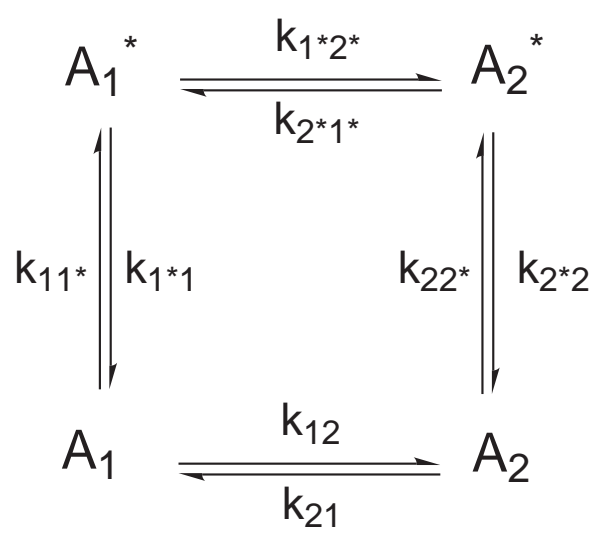

Figure 3S. Chemical process involving the smallest number of interconverting states that can be observed by FCS: An isomerization occurs between the ground states $\mathcal{A}_{1}$ and $\mathcal{A}_{2}$, and between the excited states $\mathcal{A}_{1}^{\star}$ and $\mathcal{A}_{2}^{\star}$. 
a)

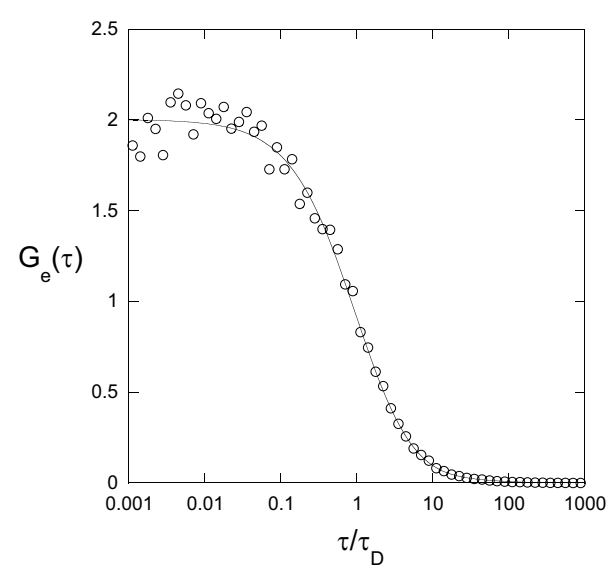

c)

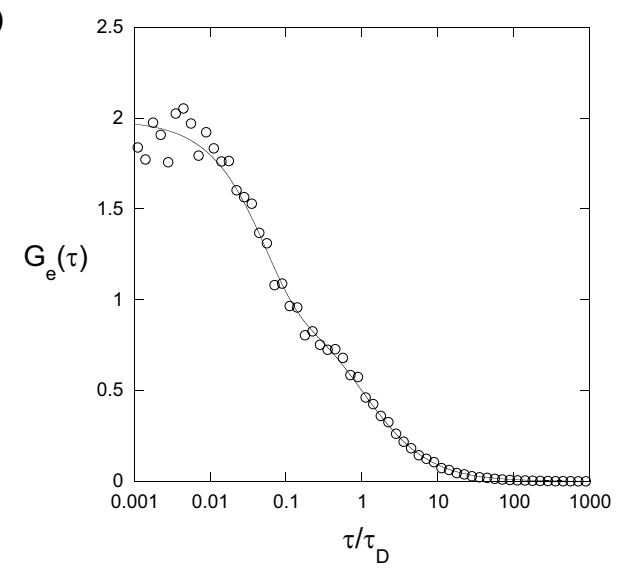

b)

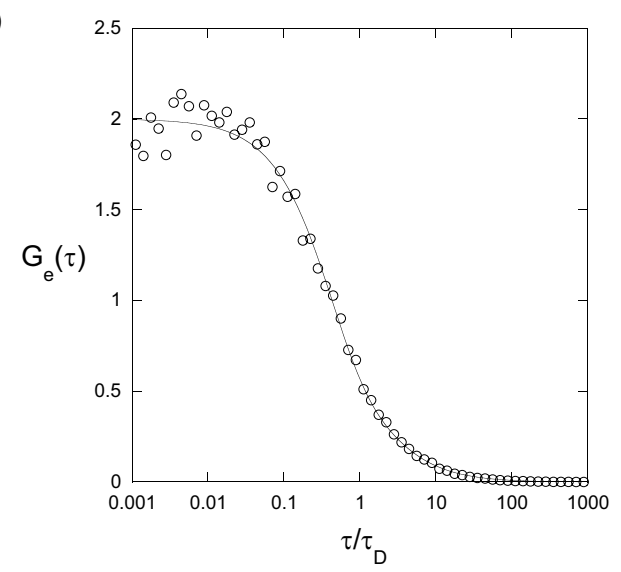

d)

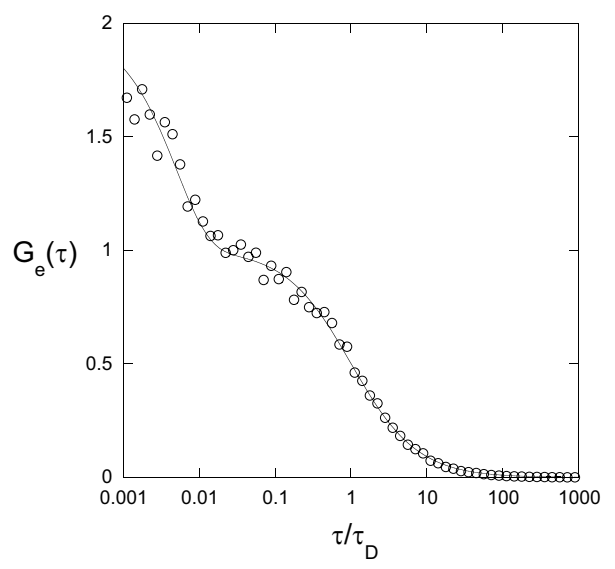

Figure 4S. Evaluation of the reliability of the parameter values that can be extracted from FCS autocorrelation curves by using Eq.(9). We used Eq.(3) to generate FCS autocorrelation curves $G_{f}(\tau)$ with $\bar{N}=1, \omega=10, \bar{H}=K$, and $Q=\infty$ for the following $k \tau_{D}$ values: $10^{-3}, 10^{-2}, 10^{-1}, 1,10^{1}, 10^{2}, 10^{3}$. Then we introduced some noise in the calculated autocorrelation functions to simulate "experimental" autocorrelation functions $G_{e}(\tau)$ (markers) by adopting the typical noise level that was observed during our present FCS measurements $\left(-0.1<\frac{G_{e}(\tau)-G_{f}(\tau)}{G_{f}(\tau)}<0.1\right)$. We finally used Eq.(9) to fit the simulated experimental data in the $10^{-3}-10^{3}$ range for $\frac{\tau}{\tau_{D}}$ by using $k \tau_{D}, \bar{N}$, and $\frac{K}{\bar{H}}$ as floatting parameters (solid line). We only present the autocorrelation curves that gave satisfactory results: $10^{-1}$ (a), $1(\mathbf{b}), 10^{1}$ (c), $10^{2}(\mathbf{d})$. 
Table 1S. Derivation of the intrinsic relaxation times $\tau_{i j}$ associated to the six chemical reactions displayed in Figure 7b: $\tau_{12}=\left[k_{12}+k_{21}(\bar{H}+\overline{O H})\right]^{-1}, \tau_{13}=\left[k_{13}+k_{31}(\bar{H}+\bar{A})\right]^{-1}, \tau_{23}=\left[k_{32}+\right.$ $\left.k_{23}(\overline{A H}+\overline{O H})\right]^{-1}, \tau_{13^{\prime}}=\left[k_{13^{\prime}}+k_{3^{\prime} 1}(\bar{H}+\bar{Y})\right]^{-1}, \tau_{23^{\prime}}=\left[k_{3^{\prime} 2}+k_{23^{\prime}}(\overline{Y H}+\overline{O H})\right]^{-1}, \tau_{33^{\prime}}=\left[k_{33^{\prime}}(\bar{A}+\right.$ $\left.\overline{Y H})+k_{3^{\prime} 3}(\overline{A H}+\bar{Y})\right]^{-1} ; \mathrm{p} K_{a}(\mathcal{A H} / \mathcal{A})=5 ; k_{12}=2.510^{-5} \mathrm{~s}^{-1}[15] ; k_{21}=1.410^{11} \mathrm{M}^{-1} \mathrm{~s}^{-1}[15] ;$ $k_{31}=k_{3^{\prime} 1}=k_{23}=k_{23^{\prime}}=k_{33^{\prime}}=10^{10} \mathrm{M}^{-1} \mathrm{~s}^{-1} ; C<100 \mathrm{nM}$. Case 1: $\mathrm{p} H$ is fixed by dilution of $\mathcal{Y H}$ $\left(\mathrm{p} K_{a}(\mathcal{Y H} / \mathcal{Y})=0\right)$ into the $\mathcal{A}$ aqueous solution; Case 2: The $\mathcal{Y H}$ buffer $\left(\mathrm{p} K_{a}(\mathcal{Y H} / \mathcal{Y})=5\right)$ at $0.1 \mathrm{M}$ fixes the $\mathrm{p} H$ of the $\mathcal{A}$ aqueous solution.

\begin{tabular}{lccccccccc}
\hline $\mathrm{p} H$ & $\begin{array}{c}\tau_{12} \\
(\mu s)\end{array}$ & $\begin{array}{c}\tau_{13} \\
(\mu s)\end{array}$ & $\begin{array}{c}\tau_{23} \\
(m s)\end{array}$ & $\begin{array}{c}\tau_{13^{\prime}}(\mathbf{1}) \\
(p s)\end{array}$ & $\begin{array}{c}\tau_{23^{\prime}}(\mathbf{1}) \\
(m s)\end{array}$ & $\begin{array}{c}\tau_{33^{\prime}}(\mathbf{1}) \\
(m s)\end{array}$ & $\begin{array}{c}\tau_{13^{\prime}}(\mathbf{2}) \\
(n s)\end{array}$ & $\begin{array}{c}\tau_{23^{\prime}}(\mathbf{2}) \\
(n s)\end{array}$ & $\begin{array}{c}\tau_{33^{\prime}}(\mathbf{2}) \\
(n s)\end{array}$ \\
\hline & & & & & & & & & \\
4 & 0.07 & 0.91 & 1.1 & 100 & $>1$ & $>1$ & 11 & 1.1 & 1 \\
4.5 & 0.23 & 2.40 & 1.3 & 100 & $>1$ & $>1$ & 4.2 & 1.3 & 1 \\
5 & 0.71 & 4.99 & 1.9 & 100 & $>1$ & $>1$ & 2.0 & 2.0 & 1 \\
5.5 & 2.26 & 7.55 & 3.5 & 100 & $>1$ & $>1$ & 1.3 & 4.2 & 1 \\
6 & 7.07 & 9.02 & 5.0 & 100 & $>1$ & $>1$ & 1.1 & 11 & 1 \\
& & & & & & & & & \\
\hline
\end{tabular}

Table 2S. Evaluation of the reliability of the parameter values that can be extracted from FCS autocorrelation curves by using Eq.(9). We used Eq.(3) to generate FCS autocorrelation curves $G_{f}(\tau)$ with $\bar{N}=1, \omega=10, \bar{H}=K$, and $Q=\infty$ for the following $k \tau_{D}$ values: $10^{-3}, 10^{-2}, 10^{-1}, 1,10^{1}, 10^{2}$, $10^{3}$. Then we introduced some noise in the calculated autocorrelation functions to simulate "experimental" autocorrelation functions $G_{e}(\tau)$ by adopting the typical noise level that was observed during our present FCS measurements $\left(-0.1<\frac{G_{e}(\tau)-G_{f}(\tau)}{G_{f}(\tau)}<0.1\right)$. We finally used Eq.(9) to fit the simulated experimental data in the $10^{-3}-10^{3}$ range for $\frac{\tau}{\tau_{D}}$ by using $k \tau_{D}, \bar{N}$, and $\frac{K}{\bar{H}}$ as floatting parameters (Levenberg-Marquardt algorithm implemented in Kaleidagrah with a $0.1 \%$ allowable error starting from a same set of initial values: $k \tau_{D}=\bar{N}=\frac{K}{\bar{H}}=1$ ). We only present the results that were obtained for the following $k \tau_{D}$ values: $10^{-1}, 1,10^{1}, 10^{2}$. The other values of $k \tau_{D}$ led to nonreliable results.

\begin{tabular}{clccc}
$k \tau_{D}$ (theor $)$ & $k \tau_{D}($ fit $)$ & $\bar{N}$ & $\frac{K}{\bar{H}}$ & Linear Correlation Coefficient $R$ \\
\hline & & & & \\
$10^{-1}$ & $9.5110^{-2}$ & 1.19 & 1.39 & 0.996 \\
1 & 0.960 & 1.02 & 1.03 & 0.997 \\
$10^{1}$ & 9.44 & 1.00 & 0.990 & 0.997 \\
$10^{2}$ & 98.3 & 0.996 & 0.974 & 0.997 \\
\hline
\end{tabular}




\section{References}

[1] Krichevsky, O; Bonnet, G. Rep. Prog. Phys., 2002, 65, 251-297.

[2] Rigler, R.; Mets, U.; Widengren, J.; Kask, P. Eur. J. Biophys., 1993, 22, 169-175.

[3] Widengren, J.; Mets, U.; Rigler, R. J. Phys. Chem., 1995, 99, 13368-13379.

[4] Mets, U.; Widengren, J.; Rigler, R. Chem.Phys., 1997, 218, 191-198.

[5] C. R. Cantor and P. R. Schimmel, Biophysical Chemistry, Part II, Freeman, New York, 1980.

[6] In a closed system containing a $\mathcal{A}_{i}$ solution that is homogeneously submitted to a two-photon excitation, the equation governing the relaxation dynamics is:

$$
\frac{d \overline{A_{i}^{\star}}}{d t}=\frac{1}{2} \delta_{2} I^{2}\left(A_{i, t o t}-\overline{A_{i}^{\star}}\right)-\frac{1}{\tau_{i}} \overline{A_{i}^{\star}}
$$

where $A_{i, t o t}$ designates the total concentration in $\mathcal{A}_{i}$. Upon neglecting the relaxation occurring during the duration $\delta \vartheta$ of the impulsion of the pulsed laser, the amount of excited state formed from the ground state after $\delta \vartheta$ is:

$$
\overline{A_{i}^{\star}}(\delta \vartheta)=A_{i, t o t}\left(1-e^{-\frac{1}{2} \delta_{2} I^{2} \delta \vartheta}\right)
$$

For a beam of a pulsed laser delivering at $800 \mathrm{~nm} \delta \vartheta=100 \mathrm{fs}$-wide impulsions every $T=13,9 \mathrm{~ns}$ at $1 \mathrm{~W}$ power on a solution containing an absorbing $\mathcal{A}_{i}$ characterized by $\delta_{2, i}$, one finds:

$$
\overline{A_{i}^{\star}}(\delta \vartheta) \simeq \frac{1}{2} \delta_{2} I^{2} \delta \vartheta A_{i, t o t} \sim 10^{-3} A_{i, t o t}
$$

At longer times than $\delta \vartheta$, relaxation of the excited state is first-order with a decay time $\tau$ in the ns range:

$$
\overline{A_{i}^{\star}}(t)=\overline{A_{i}^{\star}}(\delta \vartheta) e^{-t / \tau_{i}}
$$

Under assumption that $\tau_{i} \ll T$, the average concentration of excited molecules $\mathcal{A}_{i}^{\star}$ during $T$, $<A_{i}^{\star}>_{T}$, is:

$$
\frac{<A_{i}^{\star}>_{T}}{A_{i, t o t}} \simeq \frac{\overline{A_{i}^{\star}}(\delta \vartheta) \tau_{i}}{T} \simeq 7 \cdot 10^{-5}
$$

[7] T. Förster, Z. Elektrochem., 1950, 54, 42-46.

[8] a) A. Weller, Z. Elektrochem., 1952, 56, 662-668; b) A. Weller, Z. physik. Chem. Neue Folge, 1955, 3, 238-254; c) A. Weller, Z. physik. Chem. Neue Folge, 1958, 17, 224-245.

[9] S. G. Schulman, Molecular Luminescence Spectroscopy, Methods and Applications, Part 2, WileyInterscience, New York, Chichester, Brisbane, Toronto, Singapore, 1988.

[10] Magde, D.; Elson, E. L.; Webb, W. W. Phys. Rev. Lett., 1972, 11, 705-708.

[11] Elson, E. L.; Magde, D. Biopolymers, 1974, 13, 1-27. 
[12] Magde, D.; Elson, E. L.; Webb, W. W. Biopolymers, 1974, 13, 29-61.

[13] Lamb, D. C.; Schenk, A.; Röcker, C.; Scalfi-Happ, C.; Nienhaus, G. U. Biophys. J., 2000, 79, $1129-1138$.

[14] Bismuto, E.; Gratton, E.; Lamb, D. C. Biophys. J., 2001, 81, 3510-3521.

[15] M. Eigen, Angew. Chem., 1963, 75, 489-508. 